\title{
Coulomb effects on the formation of proton halo nuclei
}

\author{
Yu-Jie Liang ${ }^{1,2,3}$, Yan-Song Li ${ }^{4}$, Fu-Guo Deng ${ }^{1,2,3}$, Xi-Han Li ${ }^{1,2,3}$, Bao-An \\ Bian $^{1,2,3}$, Feng-Shou Zhang ${ }^{1,2,3}$, Zu-Hua Liu ${ }^{1,2,3,5}$, and Hong-Yu Zhou ${ }^{1,2,3 *}$ \\ 1 The Key Laboratory of Beam Technology and Material Modification of Ministry of Education, \\ Beijing Normal University, Beijing 100875, People's Republic of China \\ ${ }^{2}$ Institute of Low Energy Nuclear Physics, and Department of Material Science and Engineering, \\ Beijing Normal University, Beijing 100875, People's Republic of China \\ 3 Beijing Radiation Center, Beijing 100875, People's Republic of China \\ ${ }^{4}$ Department of Physics, Tsinghua University, Beijing 100084, People's Republic of China \\ ${ }^{5}$ China Institute of Atomic Energy, Beijing 102413, People's Republic of China
}

(Dated: August 6, 2021)

\begin{abstract}
The exotic structures in the $2 s_{1 / 2}$ states of five pairs of mirror nuclei ${ }^{17} \mathrm{O}-{ }^{17} \mathrm{~F},{ }^{26} \mathrm{Na}_{-}{ }^{26} \mathrm{P},{ }^{27} \mathrm{Mg}_{-}{ }^{27} \mathrm{P}$, ${ }^{28} \mathrm{Al}_{-}^{28} \mathrm{P}$ and ${ }^{29} \mathrm{Si}^{29} \mathrm{P}$ are investigated with the relativistic mean-field (RMF) theory and the singleparticle model (SPM) to explore the role of the Coulomb effects on the proton halo formation. The present RMF calculations show that the exotic structure of the valence proton is more obvious than that of the valence neutron of its mirror nucleus, the difference of exotic size between each mirror nuclei becomes smaller with the increase of mass number A of the mirror nuclei and the ratios of the valence proton and valence neutron root-mean-square (RMS) radius to the matter radius in each pair of mirror nuclei all decrease linearly with the increase of A. In order to interpret these results, we analyze two opposite effects of Coulomb interaction on the exotic structure formation with SPM and find that the contribution of the energy level shift is more important than that of the Coulomb barrier for light nuclei. However, the hindrance of the Coulomb barrier becomes more obvious with the increase of A. When A is larger than 34, Coulomb effects on the exotic structure formation will almost become zero because its two effects counteract with each other.
\end{abstract}

PACS numbers: 21.10.Gv, 21.60.Jz, 21.10.Dr

\section{INTRODUCTION}

Nuclear halo is a kind of exotic structures in which the nuclear matter distribution extends to large radii because of its weakly bound character. Halo nuclei have been extensively investigated both experimentally and theoretically for decades 11, 2, 3, 4, 5, 6, 7, 8, 9, 10, 11, 12, 13, 14, 15, 16, 17, 18, 19, 20, 21, 22. . However, up to date most of the halo nuclei confirmed are the neutron halo. The proton halos observed are rather scarce. Since ${ }^{8} \mathrm{~B}$ and ${ }^{17} \mathrm{Ne}$ are predicted as proton halos [23, 24, 25, 26], the first excited state of ${ }^{17} \mathrm{~F}[15,27]$ and proton-rich isotopes $\mathrm{P}$ and $\mathrm{S}$ [12, 13, 28, 29, 30] are predicted as the proton halos and some of them are probed experimentally.

There is a popular opinion that it is more difficult to form proton halo because the Coulomb barrier hinders the proton penetrating into the out region of nucleus. However, this may be not true actually in some cases. As will be seen below, for lighter nuclei the proton halo is easier to occur as compared to neutron halo due to the Coulomb interaction. The Coulomb interaction has two effects on the formation of nuclear halo. One of these effects is that it makes the energy level shift closer to the Fermi level thus facilitating the penetration of the valence proton beyond the range of nuclear force. On the other hand, the Coulomb barrier of the proton hinders the formation of halo structure. In the case of lighter nuclei, the former effect is more important than the later one.

Because nuclear force is nearly charge-independent, the structure difference between mirror nuclei should mainly come form the effects of the Coulomb interaction. Therefore, the mirror nuclei in the neutron-rich side would act as a useful reference system to explore the role of the Coulomb interaction on the exotic structure formation. For this purpose, in this work we investigate the structures of five pair of mirror nuclei ${ }^{17} \mathrm{O}_{-}{ }^{17} \mathrm{~F},{ }^{26} \mathrm{Na}_{-}{ }^{26} \mathrm{P},{ }^{27} \mathrm{Mg}-{ }^{27} \mathrm{P}$, ${ }^{28} \mathrm{Al}-{ }^{28} \mathrm{P}$ and ${ }^{29} \mathrm{Si}^{29} \mathrm{P}$ which are all in the $2 s_{1 / 2}$ state in the framework of RMF. Combining with the RMF results of the $2 s_{1 / 2}$ states of ${ }^{15} \mathrm{O}-{ }^{15} \mathrm{~N}$ and ${ }^{21} \mathrm{Ne}^{21} \mathrm{Na}$ [21], we find that for the seven pairs of mirror nuclei, the exotic structure of proton-rich nucleus is more obvious than that of its mirror nucleus, the difference of exotic size between the mirror nuclei becomes smaller with the increase of mass number A of the nucleus, both the ratios of the valence proton and the valence neutron root-mean-square (RMS) radius to the matter RMS radius in each pair of mirror nuclei decrease linearly with the increase of mass number A. In addition, in order to interpret the results above, we calculate in detail

*Email: zhy@bnu.edu.cn 
two opposite effects of energy level shifted and the Coulomb barrier hindrance on the exotic structure formation by means of the single particle model (SPM) and find that the contribution of the energy level shift is more important than that of Coulomb barrier when $\mathrm{A}$ is small. However, the hindrance of Coulomb barrier becomes more obvious with the increase of $\mathrm{A}$. When $\mathrm{A}$ is larger than 39, Coulomb effects on the exotic structure formation will almost become zero because its two effects counteract with each other.

\section{THE RMF RESULTS AND DISCUSSIONS}

As the relativistic mean-field (RMF) theory is a standard method for describing properties of the spherical nuclei and some deformed nuclei and its details can been found elsewhere such as Refs. 31, 32, 33, 34, 35, 36, 37, 38, 39, 40, 41, 42, 43, 44, 45, 46, 47], here we only describe the outline of the theory. For a system with the interacting nucleons, $\sigma, \omega$, and $\rho$ mesons and photons, the Lagrangian density is written as

$$
\begin{aligned}
\mathcal{L}= & \bar{\psi}\left[i \gamma^{\mu} \partial_{\mu}-m-g_{\sigma} \sigma-g_{\omega} \gamma^{\mu} \omega_{\mu}-g_{\rho} \gamma^{\mu} \vec{\tau} \cdot \vec{\rho}_{\mu}\right. \\
& \left.-e \gamma^{\mu} \frac{1-\tau_{3}}{2} A_{\mu}\right] \psi+\frac{1}{2} \partial^{\mu} \sigma \partial_{\mu} \sigma-\frac{1}{2} m_{\sigma}^{2} \sigma^{2} \\
& -\frac{1}{3} g_{2} \sigma^{3}-\frac{1}{4} g_{3} \sigma^{4}-\frac{1}{4} \omega^{\mu \nu} \omega_{\mu \nu}+\frac{1}{2} m_{\omega}^{2} \omega^{\mu} \omega_{\mu} \\
& -\frac{1}{4} \vec{\rho}^{\mu \nu} \cdot \vec{\rho}_{\mu \nu}+\frac{1}{2} m_{\rho}^{2} \vec{\rho}^{\mu} \cdot \vec{\rho}_{\mu}-\frac{1}{4} A^{\mu \nu} A_{\mu \nu},
\end{aligned}
$$

where

$$
\begin{aligned}
\omega^{\mu \nu} & =\partial^{\mu} \omega^{\nu}-\partial^{\nu} \omega^{\mu}, \\
A^{\mu \nu} & =\partial^{\mu} A^{\nu}-\partial^{\nu} A^{\mu}, \\
\vec{\rho}^{\mu \nu} & =\partial^{\mu} \vec{\rho}^{\nu}-\partial^{\nu} \vec{\rho}^{\mu}-2 g_{\rho}\left(\vec{\rho}^{\mu} \times \vec{\rho}^{\nu}\right),
\end{aligned}
$$

The nucleon field and the rest mass are denoted as $\psi$ and $m$, respectively. The meson fields and their masses are denoted by $\sigma, \omega, \rho$ and $m_{\sigma}, m_{\omega}, m_{\rho}$, respectively. The photon field $A_{\mu}$ produces the electromagnetic interaction, and $e$ is its coupling constant. $g_{\sigma}, g_{\omega}, g_{\rho}$ are the coupling constants between the mesons and nucleons respectively, $g_{2}$ and $g_{3}$ are the non-linear coupling constants of the $\sigma$ meson. $\tau_{3}$ is the third component of the isospin Pauli matrices, i.e., $\tau_{3}|n\rangle=|n\rangle$ and $\tau_{3}|p\rangle=-|p\rangle$. Using Euler-Lagrang equation, a set of coupled equations for nucleons, mesons and photons can be obtained from the Lagrangian density function, which can been solved self-consistently by iteration under the mean-field approximation. After the final solutions are obtained, some quantities we need, such as the binding energy, single-particle levels, root-mean-square (RMS) radii of neutron and proton density distributions, and so on, can be calculated from the wave functions. In the effective lagrangian density function of the relativistic mean-field $m$ is the mean value of the rest masses of proton and neutron, $e$ satisfies $e^{2} / 4 \pi=1 / 137$ and $m_{\sigma}, m_{\omega}$, $m_{\rho}, g_{\sigma}, g_{\omega}, g_{\rho}, g_{2}, g_{3}$ are free parameters. There are several well-tested nonlinear RMF parameter sets NL1, NL2, NL3 and NL-SH which were obtained by fitting the experimental observables, such as the binding energies and radii of the nuclei [31, 32, 33, 34, 35, 36, 37, 38, 39, 40, 41, 42, 43, 44, 45, 46, 47]. For usual RMF calculation there are two methods using spherical coordinate system and cylindrical coordinate system, which are used in calculations of spherical nuclei and reformed nuclei, respectively.

In this work five pairs of mirror nuclei ${ }^{17} \mathrm{~F}-{ }^{17} \mathrm{O},{ }^{26} \mathrm{P} \_{ }^{26} \mathrm{Na},{ }^{27} \mathrm{P} \_{ }^{27} \mathrm{Mg},{ }^{28} \mathrm{P} \_{ }^{28} \mathrm{Al}$ and ${ }^{29} \mathrm{P} \_{ }^{29} \mathrm{Si}$ and their core nuclei ${ }^{16} \mathrm{O},{ }^{25} \mathrm{Na},{ }^{25} \mathrm{Si},{ }^{26} \mathrm{Mg},{ }^{26} \mathrm{Si},{ }^{27} \mathrm{Al},{ }^{27} \mathrm{Si},{ }^{28} \mathrm{Si}$ are calculated by RMF. In the calculations the parameter set NL1 [34, 36 ] is chosen for ${ }^{16} \mathrm{O},{ }^{17} \mathrm{~F}$, and ${ }^{17} \mathrm{O}$, and NL3 [41, 45] are chosen for other nuclei, and the Pauli blocking effects are considered. In addition, all nuclei are considered as spherical, which means the deformation effect is omitted to simplify the calculations.

The RMF calculation results for the $2 s_{1 / 2}$ states of five pairs of mirror nuclei and their relative core nuclei are listed in Table I. $B_{\text {exp }}$ and $B_{\text {the }}$ are the experimental and the calculated binding energies, respectively. $R_{n}, R_{p}, R_{m}$ and $R_{L N}$ denote the calculated RMS radii of neutron, proton, matter and the last valence nucleon density distributions, respectively, $\varepsilon_{L N}$ is the single-particle energy of the last nucleon, $R_{L N} / R_{m}$ is the ratio of the valence nucleon RMS radius to the matter radius, and $\left|R_{n}-R_{p}\right|$ represents the difference between the proton and the neutron RMS radii. In Table I] $B_{\text {exp }}$ are taken from Ref.[48], in which $B_{\text {exp }}$ of the nucleus ${ }^{26} \mathrm{P}$ is the estimated value (denoted as \#) by Audi and Wapstra because its experimental binding energy is unknown. The density distributions of neutron, proton, matter and the valence nucleus in the mirror nuclei ${ }^{17} \mathrm{~F}-{ }^{17} \mathrm{O}$ which are calculated by the RMF, are shown in Fig. 1 . Here the normalized density distributions $\rho(r)$, which satisfies $\int_{0}^{+\infty} \rho(r) r^{2} d r=1$, is shown in order to compare the 
TABLE I: The RMF results of five pairs of mirror nuclei ${ }^{17} \mathrm{O}_{-}{ }^{17} \mathrm{~F},{ }^{26} \mathrm{Na}_{-}{ }^{26} \mathrm{P},{ }^{27} \mathrm{Mg}_{-}{ }^{27} \mathrm{P},{ }^{28} \mathrm{Al}_{-}{ }^{28} \mathrm{P}$ and ${ }^{29} \mathrm{Si}^{-29} \mathrm{P}$ and their core nuclei.

\begin{tabular}{cccccccccc}
\hline \hline & $B$ exp. $(\mathrm{MeV})$ & $B_{\text {the. }}(\mathrm{MeV})$ & $R_{n}(\mathrm{fm})$ & $R_{p}(\mathrm{fm})$ & $R_{m}(\mathrm{fm})$ & $R_{L N}(\mathrm{fm})$ & $\varepsilon_{L N}(\mathrm{MeV})$ & $R_{L N} / R_{m}\left|R_{n}-R_{p}\right|(\mathrm{fm})$ \\
\hline${ }^{16} \mathrm{O}$ & 127.62 & 127.15 & 2.64 & 2.66 & 2.65 & & & & 0.02 \\
${ }^{17} \mathrm{O}$ & 130.89 & 130.20 & 2.90 & 2.67 & 2.79 & 4.40 & -3.49 & 1.58 & 0.23 \\
${ }^{17} \mathrm{~F}$ & 127.72 & 127.18 & 2.66 & 2.99 & 2.84 & 4.77 & -0.33 & 1.68 & 0.33 \\
${ }^{25} \mathrm{Na}$ & 202.53 & 199.03 & 2.94 & 2.80 & 2.88 & & & & 0.14 \\
${ }^{26} \mathrm{Na}$ & 208.15 & 205.71 & 3.05 & 2.82 & 2.95 & 4.01 & -7.04 & 1.36 & 0.23 \\
${ }^{25} \mathrm{Si}$ & 187.01 & 183.88 & 2.80 & 3.03 & 2.93 & & & & 0.23 \\
${ }^{26} \mathrm{P}$ & $187.15 \#$ & 185.45 & 2.82 & 3.13 & 3.00 & 4.29 & -1.67 & 1.43 & 0.31 \\
${ }^{26} \mathrm{Mg}$ & 216.68 & 211.71 & 2.92 & 2.86 & 2.89 & & & & 0.06 \\
${ }^{27} \mathrm{Mg}$ & 223.12 & 219.29 & 3.02 & 2.87 & 2.96 & 3.94 & -7.97 & 1.33 & 0.15 \\
${ }^{26} \mathrm{Si}$ & 206.05 & 201.55 & 2.84 & 2.97 & 2.91 & & & & 0.13 \\
${ }^{27} \mathrm{P}$ & 206.94 & 204.27 & 2.88 & 3.10 & 3.00 & 4.17 & -2.53 & 1.39 & 0.22 \\
${ }^{27} \mathrm{Al}$ & 224.95 & 219.78 & 2.90 & 2.88 & 2.89 & & & & 0.02 \\
${ }^{28} \mathrm{Al}$ & 232.68 & 228.32 & 3.00 & 2.90 & 2.95 & 3.88 & -8.81 & 1.32 & 0.10 \\
${ }^{27} \mathrm{Si}$ & 219.36 & 214.12 & 2.85 & 2.94 & 2.90 & & & & 0.09 \\
${ }^{28} \mathrm{P}$ & 221.42 & 217.19 & 2.87 & 3.05 & 2.97 & 4.08 & -3.20 & 1.37 & 0.18 \\
${ }^{28} \mathrm{Si}$ & 236.54 & 230.78 & 2.88 & 2.92 & 2.90 & & & & 0.04 \\
${ }^{29} \mathrm{Si}$ & 245.01 & 240.25 & 2.98 & 2.93 & 2.96 & 3.83 & -9.72 & 1.29 & 0.05 \\
${ }^{29} \mathrm{P}$ & 239.29 & 234.68 & 2.90 & 3.03 & 2.97 & 4.00 & -4.02 & 1.35 & 0.13 \\
\hline \hline
\end{tabular}

relative distributions of protons and neutrons. Based on Table $\Pi$ and Fig. 1, the following discussions are made and some important conclusions are obtained.

First, for the double magic nucleus ${ }^{16} \mathrm{O}$ and its neighbor nuclei ${ }^{17} \mathrm{O}$ and ${ }^{17} \mathrm{~F}$, the difference between the theoretical binding energy $B_{\text {the }}$ and the corresponding experimental value $B_{\text {exp }}$ is very small, and $B_{t h e}$ is at most $0.5 \%$ off. For other four pairs of mirror nuclei and their core nuclei, there are good agreement between their $B_{t h e}$ and $B_{\text {exp }}$, in which for four pairs of mirror nuclei, $B_{\text {the }}$ is at most $2 \%$ off, and for other core nuclei $B_{\text {the }}$ is at most $2.5 \%$ off. These results show that it is reasonable and reliable for us to use the spherical RMF theory to describes the properties and structures of the nuclei considered.

Second, it can be seen from Fig. 1 that there are long tails in the density distribution of the valence proton of ${ }^{17} \mathrm{~F}$ and the valence neutron of ${ }^{17} \mathrm{O}$, compared with their core nucleus. The similar distributions also exist in other four pairs of mirror nuclei. These show that all of the five pairs of the mirror nuclei have exotic structures of halo or skin.

Third, there are obvious differences in exotic size of each pair of mirror nuclei. For example, the binding energy $\varepsilon_{L N}$ of the valence proton is much lower than that of the valence neutron in its mirror nucleus, and the RMS radium $R_{L N}$ and $\left|R_{p}-R_{n}\right|$ of the valence proton is larger than that of the valence neutron in its mirror nucleus. In addition, it can also be seen from Fig. 1 that the tails in the density distribution of the valence proton of ${ }^{17} \mathrm{~F}$ are longer than that of the valence neutrons of its mirror nuclei ${ }^{17} \mathrm{O}$. The similar results occur in other four pairs of mirror nuclei. These show the exotic structure of valence proton is more obvious than that of the valence neutron of its mirror nucleus.

Fourth, it can be seen from the change of the values $R_{L N} / R_{m}$ of the five pairs of mirror nuclei in Table $\square$ that the values $R_{L N} / R_{m}$ of the valence protons and the valence neutrons in each pair of the mirror nuclei all decrease with increase of mass number A. Also, the difference of exotic size between each pair of mirror nuclei becomes smaller with the increase of mass number $\mathrm{A}$ of the nucleus. In order to compare the size of the exotic structure between mirror nuclei more clearly, we plot $R_{L N} / R_{m}$ as a function of the mass number $\mathrm{A}$ in Fig. 2. The RMF results in the $2 s_{1 / 2}$ state of the other two pairs of mirror nuclei ${ }^{15} \mathrm{O}-{ }^{15} \mathrm{~N}$ and ${ }^{21} \mathrm{Ne}-{ }^{21} \mathrm{Na}$ [21] are also shown in this figure for comparison. It can be seen from Fig.2 that the values of ${ }^{15} \mathrm{~N},{ }^{17} \mathrm{~F},{ }^{21} \mathrm{Na},{ }^{26} \mathrm{P},{ }^{27} \mathrm{P},{ }^{28} \mathrm{P}$ and ${ }^{29} \mathrm{P}$ are consistently larger than those of their respective mirror partners, and the values $R_{L N} / R_{m}$ of the valence-proton nuclei and the valence-neutron nuclei are almost in two different lines and decrease gradually with the increase of mass number A. The two linear functions obtained with the least square fit are $\left(R_{L N} / R_{m}\right)_{p}=2.16176-0.02824 A$ for the valence proton nuclei (valence proton line) and $\left(R_{L N} / R_{m}\right)_{n}=1.95596-0.02302 A$ for the valence neutron nuclei (valence neutron line), respectively. The difference between the mirror nuclei decreases with the increase of mass number $A$, so as two lines intersect at about $\mathrm{A}=39$. In addition, it is clear that the difference between the valence proton line and the valence neutron line also decreases linearly with increase of mass number A. This difference line (RMF Coulomb line) corresponds to the contribution of the pure Coulomb effect on the valence proton dispersion. 


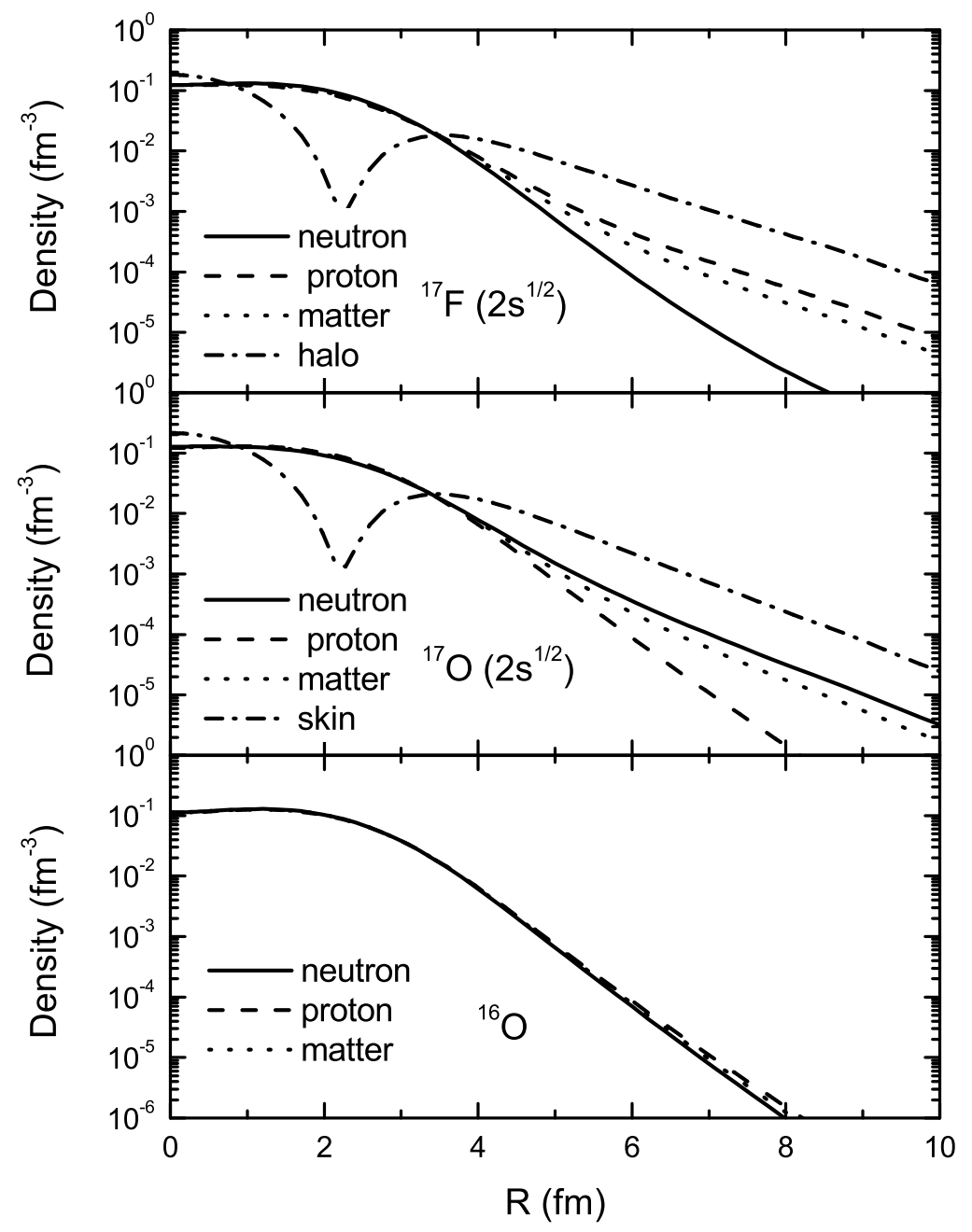

FIG. 1: The density distributions of neutron, proton, matter, and the last nucleon in ${ }^{17} \mathrm{O}-{ }^{17} \mathrm{~F}$ in the first excited state. Solid, dashed, dotted, and dash-dotted curves are the density distributions of protons, neutrons, matter and halo proton, respectively. The density distributions of their core nucleus ${ }^{16} \mathrm{O}$ is also drawn for comparing.

The above results can be explained qualitatively by the RMF calculation results. Because the nuclear force is approximately charge-independent and there are the same nuclear force interaction in each pair of mirror nuclei, the difference between the structures of mirror nuclei should only come from the Coulomb interaction. The Coulomb effects on valence proton distribution include two opposite actions. One action is that it makes the energy level shift closer to the Fermi level, which means the valence proton is easier to extend far from the nuclear potential. The other action is that the Coulomb barrier hinders the diffusion of the valence proton and formation of exotic structure. We plot the single nucleon energy levels of $2 s_{1 / 2}$ states of five pairs of the mirror nuclei in Fig. 3 and the nuclear, the Coulomb and the total potentials for ${ }^{17} \mathrm{O}-{ }^{17} \mathrm{~F}$ and ${ }^{29} \mathrm{Si}^{29} \mathrm{P}$ in Fig. 4. The potentials for the other three pairs of mirror nuclei are similar to that of ${ }^{17} \mathrm{O}-{ }^{17} \mathrm{~F}$ and ${ }^{29} \mathrm{Si}^{29}{ }^{29} \mathrm{P}$. It can be seen from Fig. 3 that the single proton energy levels in ${ }^{17} \mathrm{~F},{ }^{21} \mathrm{Na},{ }^{26} \mathrm{P},{ }^{27} \mathrm{P},{ }^{28} \mathrm{P}$ and ${ }^{29} \mathrm{P}$ are higher than the corresponding single neutron energy levels in their mirror nucleus ${ }^{17} \mathrm{O},{ }^{26} \mathrm{Na},{ }^{27} \mathrm{Mg},{ }^{28} \mathrm{Al}$ and ${ }^{29} \mathrm{Si}$, which is induced by the Coulomb interaction. It can also be seen from Fig. 3 that the energy level shifts in the $2 s_{1 / 2}$ states between mirror nuclei become larger with the increase of mass number A except ${ }^{17} \mathrm{O}$ and ${ }^{17} \mathrm{~F}$. On the other hand, the calculation results shown that the hights of Coulomb barrier increase with the increase of mass number A. The Coulomb barriers of ${ }^{17} \mathrm{~F}$ and ${ }^{29} \mathrm{P}$ are shown in Fig.5. The Coulomb barrier of ${ }^{26} \mathrm{P},{ }^{27} \mathrm{P}$, and ${ }^{28} \mathrm{P}$ lie between those of ${ }^{17} \mathrm{~F}$ and ${ }^{29} \mathrm{P}$ (not shown in the figure). The two opposite effects of Coulomb 


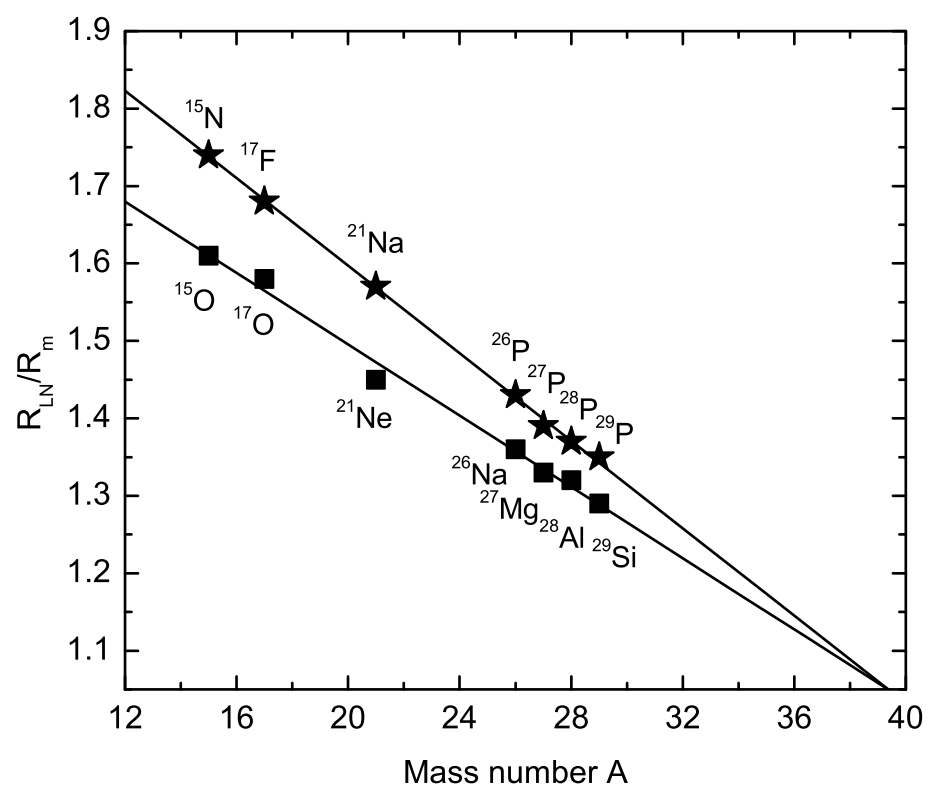

FIG. 2: The ratios of the last nucleon RMS radius to the matter one as a function of mass number in mirror nuclei. The solid stars stand for the ratios in the proton-rich nuclei and the solid squares stand for that in the neutron-rich nuclei. The results for ${ }^{15} \mathrm{O}-{ }^{15} \mathrm{~N}$ and ${ }^{21} \mathrm{Ne}^{21} \mathrm{Na}$ are taken from Ref. [21]. The lines are the least square fit for the data.

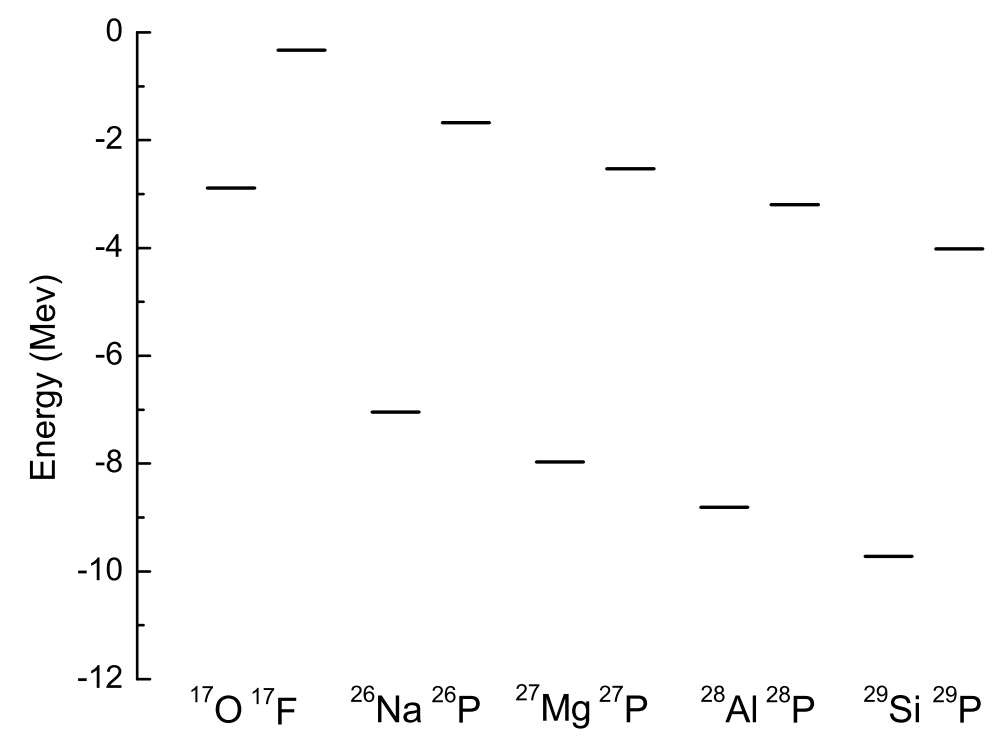

FIG. 3: The single neutron energy levels of $2 s_{1 / 2}$ states for ${ }^{17} \mathrm{O},{ }^{26} \mathrm{Na},{ }^{27} \mathrm{Mg},{ }^{28} \mathrm{Al},{ }^{29} \mathrm{Si}$ and the single proton energy levels $2 s_{1 / 2}$ states for ${ }^{17} \mathrm{~F},{ }^{26} \mathrm{P},{ }^{27} \mathrm{P},{ }^{28} \mathrm{P},{ }^{29} \mathrm{P}$.

interaction determine the contribution of Coulomb potential to the valence proton dispersion together. Although the RMF theory cannot separate quantitatively the two opposite effects of Coulomb interaction to valence proton, it can be inferred from Fig.4 and 5 that when mass number A is small, the contribution of the energy level shift is more important than that of the Coulomb barrier, but the hindrance of the Coulomb barrier becomes more obvious with the increase of $\mathrm{A}$. When $\mathrm{A}$ is about 39, Coulomb effects on the exotic structure formation will almost become zero because its two effects counteract with each other. This qualitative conclusion will be proven quantitatively in the following single-particle model (SPM) analysis. 


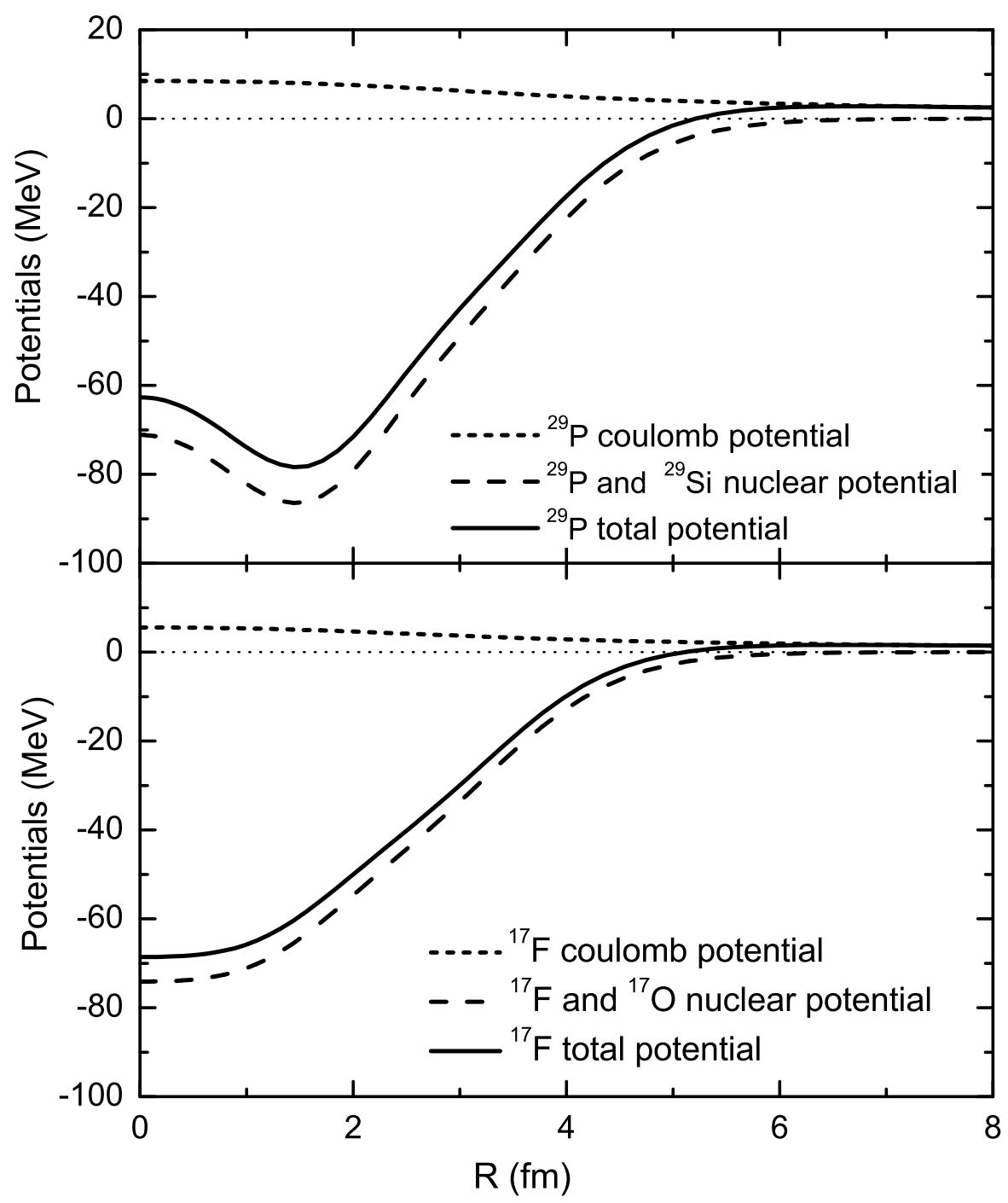

FIG. 4: The variations of the means-field potentials with the radial coordinates for ${ }^{17} \mathrm{O}-{ }^{17} \mathrm{~F}$ and ${ }^{29} \mathrm{Si}^{29}{ }^{29}$. Short-dashed, dashed and solid curves are the Coulomb potential, nuclear potential and total potential.

TABLE II: The contributions of the energy level shifted and the Coulomb barrier to exotic formation calculated with the single-particle model.

\begin{tabular}{|c|c|c|c|c|c|c|c|c|c|c|c|c|}
\hline & ${ }^{17} \mathrm{O}$ & ${ }^{17} \mathrm{~F}$ & ${ }^{21} \mathrm{Ne}$ & ${ }^{21} \mathrm{Na}$ & ${ }^{26} \mathrm{Na}$ & ${ }^{26} \mathrm{P}$ & ${ }^{27} \mathrm{Mg}$ & ${ }^{27} \mathrm{P}$ & ${ }^{28} \mathrm{Al}$ & ${ }^{28} \mathrm{P}$ & ${ }^{29} \mathrm{Si}$ & ${ }^{29} \mathrm{P}$ \\
\hline$R_{L N}(\mathrm{fm})$ & 4.27 & 5.43 & 4.40 & 5.37 & 4.19 & 4.87 & 4.10 & 4.60 & 3.98 & 4.33 & 3.93 & 4.22 \\
\hline$R_{i}(\mathrm{fm})$ & & 6.68 & & 9.49 & & 5.10 & & 2.23 & & 1.24 & & 0.98 \\
\hline$R_{d}(\mathrm{fm})$ & & -5.52 & & -8.52 & & -4.42 & & -1.73 & & -0.89 & & -0.39 \\
\hline$R_{c}(\mathrm{fm})$ & & 1.16 & & 0.97 & & 0.68 & & 0.50 & & 0.35 & & 0.29 \\
\hline
\end{tabular}

\section{THE SPM RESULTS AND DISCUSSIONS}

In the single-particle model, the nucleus is assumed to be composed of the nuclear core and the valence nucleon outside the core. The normalized single-particle radial wave function in the $(n l j)$ bound state $\phi_{n l j}(r)$ can be obtained by solving the Schrödinger equation. The potential is chosen as

$$
V(r)=V_{N}(r)+V_{C}(r),
$$




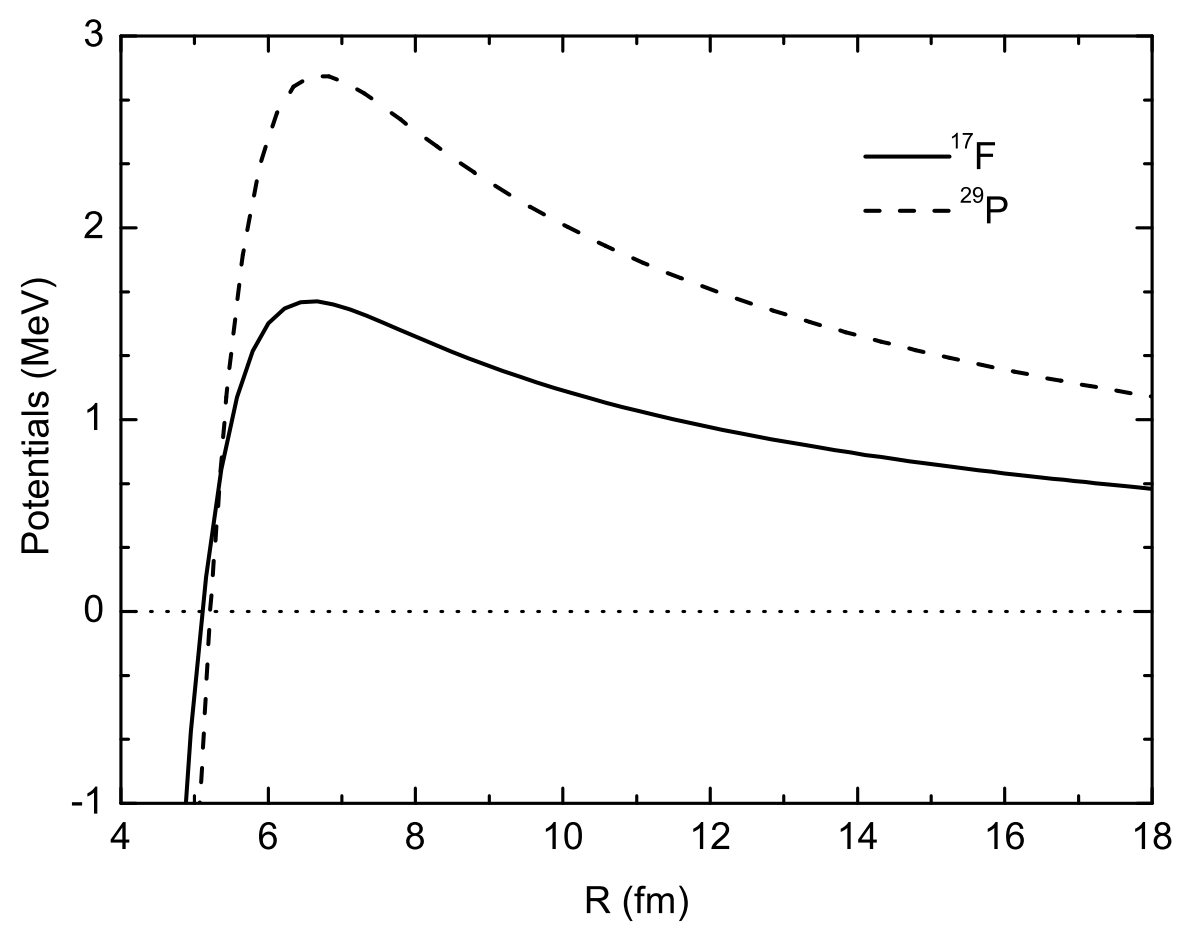

FIG. 5: The Coulomb barriers of ${ }^{17} \mathrm{~F}$ and ${ }^{29} \mathrm{P}$. The solid and dashed curves are for ${ }^{17} \mathrm{~F}$ and ${ }^{29} \mathrm{P}$, respectively.

where the nuclear potential $V_{N}(r)$ is chosen as Woods-Saxon potential

$$
V_{N}(r)=V_{0} /\left\{1+\exp \left(\frac{r-r_{0} A^{1 / 3}}{a_{0}}\right)\right\}
$$

and $V_{C}(r)$ is Coulomb potential. Here the potential depth $V_{0}$ of Woods-Saxon potential is adjusted to reproduce the valence nucleon separation energy, $r_{0}$ and $a_{0}$ are the radius and diffuseness parameter, respectively. In this work, $r_{0}$ and $a_{0}$ are chosen as the normal values $1.25 \mathrm{fm}$ and $0.65 \mathrm{fm}$, respectively. The root-mean-square (rms) radius of the distribution of the last nucleon can be obtained from the single-particle radial wave function $\phi_{n l j}(r)$ by

$$
R_{L N}=\left[\int_{0}^{\infty} r^{4} \phi_{n l j}^{2}(r) d r\right]^{1 / 2}
$$

The numerical results with the SPM are listed in Table In where $R_{i}$ denotes the increase of $R_{L N}$ induced by the energy level shifted and $R_{d}$ denotes the decrease of $R_{L N}$ induced by the Coulomb barrier. $R_{c}$ denotes the contribution of total Coulomb effect on the valence proton dispersion and $R_{c}=R_{i}+R_{d}$. We select ${ }^{17} \mathrm{O}-{ }^{17} \mathrm{~F}$ as an example to explain the details of the calculations. At first, we obtain the RMS radii of the valence nucleon with the single-particle model $\left(4.27 \mathrm{fm}\right.$ for ${ }^{17} \mathrm{O}$ and $5.43 \mathrm{fm}$ for $\left.{ }^{17} \mathrm{~F}\right)$. Next, we assume that the binding energy of ${ }^{17} \mathrm{O}$ is the same as ${ }^{17} \mathrm{~F}$ and calculate the valence neutron RMS radii for ${ }^{17} \mathrm{O}$ under this condition. The result is $10.95 \mathrm{fm}$, which is much larger than the actual value of ${ }^{17} \mathrm{O}$. This implies that the energy level shifted makes the RMS radius increase $6.68 \mathrm{fm}$ if the effects of Coulomb barrier switch off. However, the Coulomb barrier hinders the exotic formation in reality. The RMS radius of ${ }^{17} \mathrm{~F}$ is less than the result obtained by neglecting the effect of the Coulomb barrier. Therefore, the Coulomb barrier makes RMS radii decrease $5.52 \mathrm{fm}$. In Table II "_" of $R_{d}$ values shows decrease of the valence proton dispersion. It shows that two effects of the Coulomb interaction are opposite on the formation of exotic structures of valence proton, and the contributions of the energy level shifted are more important than that of the Coulomb barrier. The same calculations are applied to the other pairs of mirror nuclei. In order to show these rules more clearly, the variations of $R_{i}, R_{d}$ and $R_{c}$ with the mass numbers are given in Fig.6. It is seen from Table II and Fig.6 that the two effects of the Coulomb interaction on the valence proton dispersion are opposite, and when mass number $\mathrm{A}$ is smaller the contribution of the energy level shifted is more important than that of the Coulomb barrier hindrance. However, the hindrance of the Coulomb barrier becomes more obvious with the increase of $\mathrm{A}$, and total effect of the Coulomb interaction on the valence proton dispersion decreases linearly with increase of the mass number A of the 


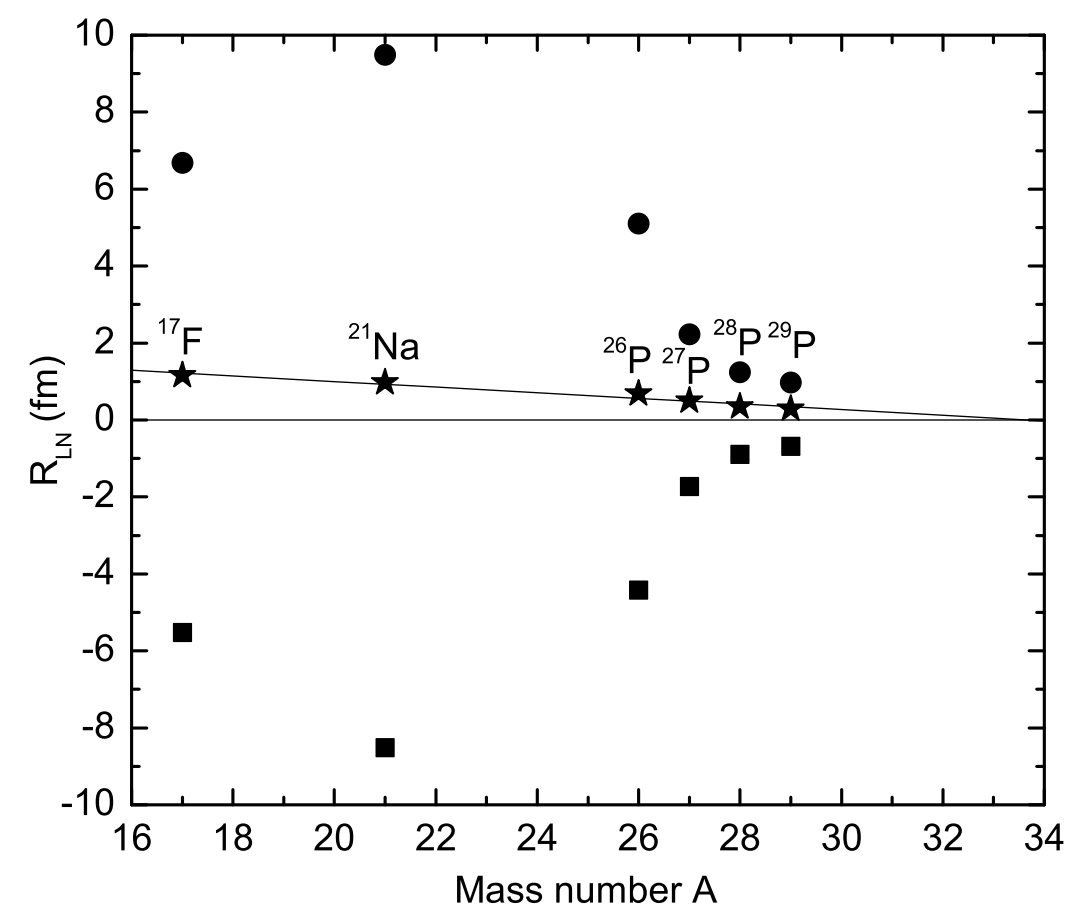

FIG. 6: The contributions of the energy level shift and the Coulomb barrier to exotic formation calculated with the singleparticle approach. The solid circles denote the increase of $R_{L N}$ induced by the energy level shifted, the solid squares denote the decrease of $R_{L N}$ induced by the Coulomb barrier and the solid stars denote the total Coulomb effect on $R_{L N}$.

nucleus, and when $\mathrm{A}$ is larger than about 34, Coulomb effects on the exotic structure formation will almost become zero because its two effects counteract with each other. These conclusions are in good agreement with those obtained with RMF although there are some differences in their values, which maybe come from the difference between the two theoretical models RMF and SPM.

\section{SUMMARY}

In summary, we have investigated the exotic structures in $2 s_{1 / 2}$ state of five pairs of mirror nuclei ${ }^{17} \mathrm{O}_{-}{ }^{17} \mathrm{~F},{ }^{26} \mathrm{Na}^{2}{ }^{26} \mathrm{P}$, ${ }^{27} \mathrm{Mg}^{27} \mathrm{P},{ }^{28} \mathrm{Al}^{28} \mathrm{P}$ and ${ }^{29} \mathrm{Si}^{-29} \mathrm{P}$ with RMF and SPM in order to explore the role of the Coulomb interaction on the proton halo formation. By analyzing the RMF results, we find that the exotic structure of valence proton is more obvious than that of the valence neutron of its mirror nucleus, the $R_{L N} / R_{m}$ values of the valence proton and the valence neutron of each pair of mirror nuclei decrease linearly with the increase of mass number A of the mirror nuclei, and the difference between the values of each pair of mirror nuclei becomes smaller linearly with the increase of mass number $\mathrm{A}$. When $\mathrm{A}$ is about 39, the difference closes zero. By analyzing quantitatively two opposite effects of the Coulomb interaction on the valence proton dispersion in some mirror nuclei with SPM, we find that the contributions of the energy level shift are more important than that of the Coulomb barrier, and the total Coulomb effect on the valence proton dispersion becomes smaller linearly with increase of the mass number A, and it closes zero when $\mathrm{A}$ is larger than 34, which means two effects of Coulomb interaction on valence proton counteract with each other. These results basically agree with the conclusions obtained by RMF. 


\section{ACKNOWLEDGMENTS}

This work is supported by the National Natural Science Foundation of China under Grant Nos 10604008 and 10435020, A foundation for the Author of National Excellent Doctoral Dissertation of China, and Beijing Education Committee Under Grant No. XK100270454.

[1] I. Tanihata, H. Hamagaki, O. Hashimoto, Y. Shida et al., Phys. Rev. Lett. 55, 2676 (1985).

[2] W. Mittig, J. M. Chouvel, W. L. Zhan, L. Bianchi et al., Phys. Rev. Lett. 59, 1889 (1987).

[3] P. G. Hansen and B. Jonson, Europhys. Lett. 4, 409 (1987).

[4] Y. Suzuki and K. Ikeda, Phys. Rev. C 38, 410 (1988).

[5] M. G. Saint-Laurent et al., Z. Phys. A 332, 457 (1989)

[6] E. Liatard et al., Europhys. Lett. 13, 495 (1990).

[7] L. Johannsen, A. S. Jensen, and P. G. Hansen, Phys. Lett. B 244, 357 (1990).

[8] G. F. Bertsch and E. Esbensen, Ann. Phys. (N. Y) 209, 327 (1991).

[9] A. C. Hayes, Phys. Lett. B 254, 15 (1991).

[10] M. V. Zhukov, B. V. Danilin, D. V. Fedorov et al., Phys. Rep. 231, 151 (1993).

[11] I. J. Thompson, J. S. Al-khalili, J. A. Tostevin, and J. M. Bang, Phys. Rev. C 47, 1364 (1993).

[12] Z. Z. Ren, B. Q. Chen, Z. Y. Ma, and G. O. Xu, Phys. Rev. C 53, R572 (1996).

[13] Z. Z. Ren, W. Mitting, and F. Sarazin, Nucl. Phys. A 652, 250 (1999).

[14] Z. H. Liu, C. J. Lin, H. Q. Zhang et al., Phys. Rev. C 64, 034312 (2001).

[15] R. Morlock et al., Phys. Rev. Lett. 79, 3827(1997).

[16] V. Guimaraes, S. Kubono et al., Nucl. Phys. A 588, 161c (1995).

[17] L. Axelsson, M. J. G. Borge et al., Phys. Rev. C 54, R1511 (1996).

[18] S. Aoyama, Phys. Rev. C 62, 034305 (2000).

[19] N. Auerbach and N. Vinh Mau, Phys. Rev. C 63, 017301 (2000).

[20] H. Y. Zhang, W. Q. Shen, Z. Z. Ren, Y. G. Ma et al., Eur. Phys. J. A 23, 11 (2005).

[21] J. G. Chen, X. Z. Cai, W. Q. Shen, Y. G. Ma et al., Eur. Phys. J. A 23, 11 (2005).

[22] Y. J. Liang, X. H. Li, F. G. Deng et al., Chin. Phys. Lett 23, 1142 (2006).

[23] K. Riisager and A. S. Jensen, Phys. Lett. B 315, 6 (1993).

[24] J. S. Al-khalili, J. A. Tostevin, and I. J. Thompson, Phys. Rev. C 54, 1843 (1996).

[25] H. Esbensen, Phys. Rev. C 53, 2007 (1996).

[26] B. J. Cole, Phys. Rev. C 58, 1866 (1998).

[27] Z. Z. Ren, A. Faessler, and A. Bobyk, Phys. Rev. C 57, 2752 (1998).

[28] B. A. Brown and P. G. Hansen, Phys. Lett. B 381, 391 (1996).

[29] B. Q. Chen, Z. Y. Ma et al., J. Phys. G: Nucl. Phys. 24, 97 (1998)

[30] A. Navin et al., Phys. Rev. Lett. 81, 5089 (1998).

[31] J. D. Walecka, Ann. Phys. 83, 491 (1974).

[32] C. J. Horowitz and B. D. Serot, Nucl. Phys. A 368, 503 (1981) .

[33] B. D. Serot and J. D. Walecka, Adv. Nucl. Phys. 16, 1 (1986) .

[34] P. G. Reinhard, M. Rufa, J. A. Maruhn, W. Greiner, and J. Friedrich, Z. Phys. 323, 13 (1986).

[35] M. Rufa, P. G. Reinhard, J. A. Maruhn, W. Greiner, and M. R. Strayer, Phys. Rev. C 38, 390 (1988).

[36] P. G. Reinhard, Rep. Prog. Phys. 52439 (1989).

[37] I. Tanihata, D. Hirata, T. Kobayashi, S. Shimoura, K. Sugimoto, and H. Toki, Phys. Lett. B 289, 261 (1992).

[38] D. Hirata, H. Toki, 1. Tanihata, and P. Ring, Phys. Lett. B 314, 168 (1993).

[39] L. S. Warrier and Y. K. Gambhir, Phys. Rev. C 49, 871 (1994).

[40] P. Ring, Prog. Part. Nucl. Phys. 37, 193 (1996).

[41] G. A. Lalazissis, J. Konig, and P. Ring, Phys. Rev. C 55, 540 (1997).

[42] B. D. Serot and J. D. Walecka, Int. J. Mod. Phys. E 6, 515 (1997).

[43] J. Meng, K. Sugawara-Tanabe, S. Yamaji, P. Ring, and A. Arima, Phys. Rev. C 58, R628 (1998).

[44] J. Meng, K. Sugawara-Tanabe, S. Yamaji, and A. Arima, Phys. Rev. C 59, 154 (1999).

[45] Z. Patyk, A. Baran, J. F. Berger, J. Decharge, J. Dobaczewski, P. Ring, and A. Sobiczewski, Phys. Rev. C 59 , 704 (1999).

[46] M. Bender, P. H. Heenen, and P. G. Reinhard, Rev. Modern Phys. 75, 121 (2003).

[47] Y. S. Li and G. L. Long, Commun. Theor. Phys. 41, 429 (2004).

[48] G. Audi and A.H. Wapstra, Nucl. Phys. A 565, 1 (1993). 